\title{
Kounis syndrome: A primary cause for the anaphylactic shock
}

In the important paper of Kuda et al. [1] published in "Cardiology Journal" it was found that vasospasm-induced coronary flow reduction is a major contributory factor in anaphylactic ventricular dysfunction. The authors concluded that during anaphylaxis the ensuing left ventricular dysfunction is attributed mainly to coronary vasoconstriction-induced myocardial ischemia. These and other experimental findings, together with clinical observations according to which the anaphylactic cardiac shock responds to acute myocardial infarction protocol treatment while fluid replacement has minimal effect might have profound clinical and therapeutic implications as far as Kounis syndrome is concerned [2,3]. The current consensus is that during anaphylactic shock, the observed myocardial damage and ventricular dysfunction is the result of leakage of plasma and volume loss due to systemic vasodilatation and increased vascular permeability, reduced venous return and depression of cardiac output due to coronary hypoperfusion. In Kounis anaphylaxis associated coronary syndrome, the myocardial dysfunction is the result of acute coronary insufficiency from coronary vasospasm or coronary thrombotic obstruction. This syndrome is caused by inflammatory mediators released during the mast cell degranulation process and platelet activation via $F_{c} \gamma$ RI, Fc $\gamma$ RII, Fc $\varepsilon$ RII, and FceRII receptors [4].

Previous animal experiments have shown that anaphylaxis induces acute myocardial ischemia, decrease in cardiac output by $90 \%$, increase in left ventricular end diastolic pressure indicating pump failure and initial increase in arterial blood pressure. The increase in left ventricular end diastolic pressure suggests that volume loss due to an increase in vascular permeability and decreased venous return are unlikely to be primary causes of the documented depression in cardiac output. The authors of this experiment definitely excluded peripheral vasodilatation as a cause of the registered anaphylactic cardiac damage [5]. Similarly, in a recent study [6], it was found that anaphylactic shock decreases cerebral blood flow more than what would be expected from severe arterial hypotension. This was attributed to direct action of anaphylactic mediators on the cerebral arterial system and not solely due to arterial hypotension.

In the clinical setting [7], a patient who was stung by multiple wasps and developed type I variant of Kounis syndrome with anaphylactic shock and myocardial ischemia was treated with $2 \mathrm{~L}$ of normal saline, adrenaline, hydrocortisone, and antihistamines, which did not have any immediate effect and the patient recovered in a later stage with vasopressors and myocardial infarction protocol treatment. In another patient with Kounis syndrome due to per-operative anaphylactic shock from gelofusine infusion [8] the recovery was achieved with intravenous antihistamines, steroids and inotropic support while treatment with metaraminol and epinephrine worsened hypotension and annihilated cardiac output.

Differentiating tissue hypoperfusion from primary tissue suppression due to mast cell mediator action on systemic arterial vasculature seems to be challenging. In anaphylactic shock, myocardial involvement due to vasospasm-induced coronary blood flow reduction manifesting as Kounis syndrome should be always considered. Clinically, combined treatment targeting the primary cause of anaphylaxis together with protection of cardiac tissue seems to be of paramount importance. More studies and more experience are needed to determine the importance of mediators of anaphylaxis in myocardial pathobiology.

Conflict of interest: none declared

\section{References}

1. Kuda Y, Kurata Y, Wang M, Tanida M, Shibamoto T. Major contribution of vasospasm-induced coronary blood flow reduction to anaphylactic ventricular dysfunction assessed in isolated blood-perfused rat heart. Cardiol J, 2014; 21: 11-17.

2. Kounis NG. Coronary hypersensitivity disorder: The Kounis syndrome. Clin Ther, 2013; 35: 563-571.

3. Sinkiewicz W, Sobański P, Bartuzi Z. Allergic myocardial infarction. Cardiol J, 2008; 15: 220-225. 
4. Kounis NG, Mazarakis A, Tsigkas G, Giannopoulos S, Goudevenos J. Kounis syndrome: a new twist on an old disease. Future Cardiol, 2011; 7: 805-824.

5. Felix SB, Baumann G, Berdel WE. Systemic anaphylaxis separation of cardiac reactions from respiratory and peripheral vascular events. Res Exp Med, 1990; 190: 239-252.

6. Davidson J, Zheng F, Tajima K et al. Anaphylactic shock decreases cerebral blood flow more than what would be expected from severe arterial hypotension. Shock, 2012; 38: 429-435.

7. Mukta V, Susmitha C, Das AK. Allergic myocardial infarction. N Am J Med Sci, 2013; 5: 157-158.

8. Shah G, Scadding G, Nguyen-Lu N et al. Peri-operative cardiac arrest with ST elevation secondary to gelofusin anaphylaxis: Kounis syndrome in the anaesthetic room. Int J Cardiol, 2013; 164: e22-26.

Nicholas G. Kounis, George D. Soufras Department of Medical Sciences, Patras Highest Institute of Education and Technology, Queen Olgas Square, 7 Aratou Street, Patras 26221, Greece, tel/fax: +302610279579, e-mail:ngkounis@otenet.gr 